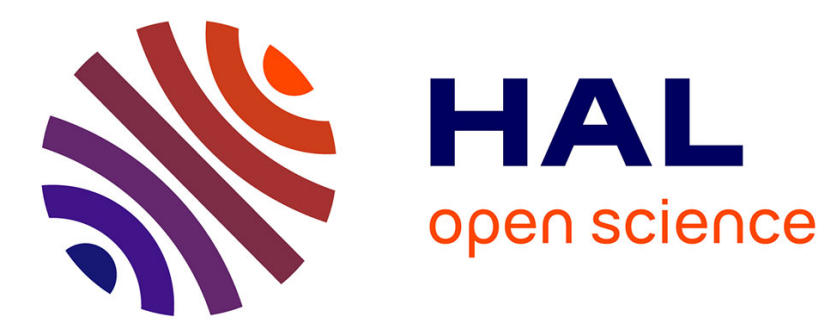

\title{
Commissioning status of the Virgo interferometer
}

T Accadia, F Acernese, F Antonucci, S Aoudia, K G Arun, P Astone, G Ballardin, F Barone, M. Barsuglia, Th S Bauer, et al.

\section{To cite this version:}

T Accadia, F Acernese, F Antonucci, S Aoudia, K G Arun, et al.. Commissioning status of the Virgo interferometer. Classical and Quantum Gravity, 2010, 27 (8), pp.0824002. 10.1088/02649381/27/8/084002 . hal-00587610

\section{HAL Id: hal-00587610 https://hal.science/hal-00587610}

Submitted on 21 Apr 2011

HAL is a multi-disciplinary open access archive for the deposit and dissemination of scientific research documents, whether they are published or not. The documents may come from teaching and research institutions in France or abroad, or from public or private research centers.
L'archive ouverte pluridisciplinaire HAL, est destinée au dépôt et à la diffusion de documents scientifiques de niveau recherche, publiés ou non, émanant des établissements d'enseignement et de recherche français ou étrangers, des laboratoires publics ou privés. 


\section{Commissioning status of the Virgo Interferometer}

T. Accadia ${ }^{12}$, F. Acernese ${ }^{12}$, F. Antonucci ${ }^{9 a}$, S. Aoudia ${ }^{15 a}$, K.G. Arun $^{11}$, P. Astone ${ }^{9 a}$, G. Ballardin ${ }^{2}$, F. Barone ${ }^{6 a c}$, M. Barsuglia ${ }^{1}$, Th.S. Bauer ${ }^{14 a}$, M.G. Beker ${ }^{14 a}$, S. Bigotta ${ }^{8 a b}$, S. Birindelli ${ }^{15 a}$, M.A. Bizouard ${ }^{11}$, M. Blom ${ }^{14 a}$, C. Boccara ${ }^{3}$, F. Bondu ${ }^{15 b}$, L.

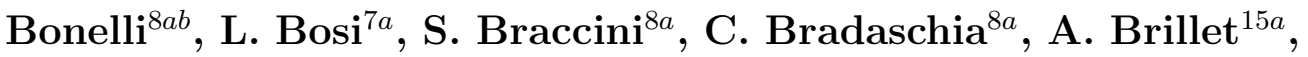
V. Brisson ${ }^{11}$, R. Budzyński ${ }^{17 b}$, T. Bulik ${ }^{17 c d}$, H.J. Bulten ${ }^{14 a b}$, D. Buskulic $^{12}$, G. Cagnoli ${ }^{4 a}$, E. Calloni ${ }^{6 a b}$, E. Campagna ${ }^{4 a c}$, B.

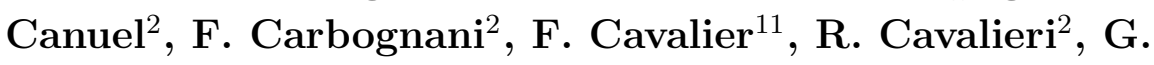
Cella $^{8 a}$, E. Cesarini ${ }^{4 c}$, E. Chassande-Mottin ${ }^{1}$, A. Chincarini ${ }^{5}$, F. Cleva $^{15 a}$, E. Coccia ${ }^{10 a b}$, C. N. Colacino ${ }^{* * 8}$, J. Colas ${ }^{2}$, A. Colla ${ }^{9 a b}$, M. Colombini ${ }^{9 b}$, A. Corsi ${ }^{9 a}$, J.-P. Coulon ${ }^{15 a}$, E. Cuoco ${ }^{2}$, S.

D'Antonio ${ }^{10 a}$, A. Dari ${ }^{7 a b}$, V. Dattilo ${ }^{2}$, M. Davier ${ }^{11}$, R. Day ${ }^{2}$, R.

De Rosa ${ }^{6 a b}$, M. del Prete ${ }^{8 a c}$, L. Di Fiore ${ }^{6 a}$, A. Di Lieto ${ }^{8 a b}$, M. Di Paolo Emilio ${ }^{10 a d}$, A. Di Virgilio ${ }^{8 a}$, A. Dietz ${ }^{*, 12}$, M. Drago ${ }^{16 c d}$, V. Fafone $^{10 a b}$, I. Ferrante ${ }^{8 a b}$, F. Fidecaro ${ }^{8 a b}$, I. Fiori ${ }^{2}$, R. Flaminio ${ }^{13}$, J.-D. Fournier ${ }^{15 a}$, J. Franc ${ }^{13}$, S. Frasca ${ }^{9 a b}$, F. Frasconi ${ }^{8 a}$, A.

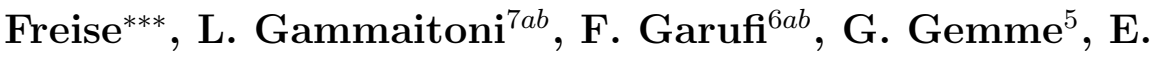

Genin $^{2}$, A. Gennai ${ }^{8 a}$, A. Giazotto ${ }^{8 a}$, R. Gouaty ${ }^{12}$, M. Granata ${ }^{1}$, C. Greverie ${ }^{15 a}$, G. Guidi ${ }^{4 a c}$, H. Heitmann ${ }^{15}$, P. Hello ${ }^{11}$, S. Hild $^{* * *}$, D. Huet ${ }^{2}$, P. Jaranowski ${ }^{17 e}$, I. Kowalska ${ }^{17 c}$, A. Królak $^{17 a f}$, P. La Penna ${ }^{2}$, N. Leroy ${ }^{11}$, N. Letendre ${ }^{12}$, T.G.F. Li ${ }^{14 a}$, M. Lorenzini ${ }^{4 a}$, V. Loriette $^{3}$, G. Losurdo ${ }^{4 a}$, J.-M. Mackowski $^{13}$, E. Majorana ${ }^{9 a}$, N. Man ${ }^{15 a}$, M. Mantovani ${ }^{8 c}$, F. Marchesoni ${ }^{7 a}$, F. Marion ${ }^{12}$, J. Marque ${ }^{2}$, F. Martelli ${ }^{4 a c}$, A.

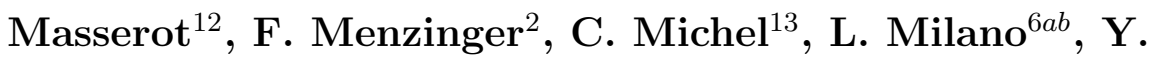
Minenkov $^{10 a}$, M. Mohan ${ }^{2}$, J. Moreau ${ }^{3}$, N. Morgado ${ }^{13}$, A. Morgia ${ }^{10 a b}$, S. Mosca ${ }^{6 a b}$, V. Moscatelli ${ }^{9 a}$, B. Mours ${ }^{12}$, I. Neri $^{7 a b}$, F. Nocera ${ }^{2}$, G. Pagliaroli ${ }^{10 a b}$, C. Palomba ${ }^{9 a}$, F. Paoletti $^{8 a, 2}$, S. Pardi ${ }^{6 a b}$, M. Parisi ${ }^{6 b}$, A. Pasqualetti ${ }^{2}$, R.

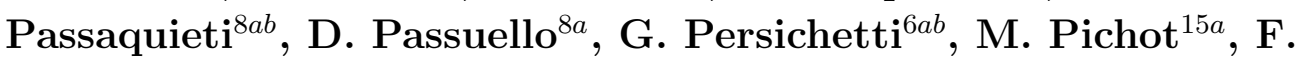
Piergiovanni $^{4 a c}$, M. Pietka ${ }^{17 e}$, L. Pinard ${ }^{13}$, R. Poggiani ${ }^{8 a b}$, M. Prato $^{5}$, G.A. Prodi ${ }^{16 a b}$, M. Punturo ${ }^{7 a}$, P. Puppo ${ }^{9 a}$, O. Rabaste ${ }^{1}$, D.S. Rabeling ${ }^{14 a b}$, P. Rapagnani ${ }^{9 a b}$, V. Re ${ }^{16 a b}$, T. Regimbau ${ }^{15 a}$, F. Ricci ${ }^{9 a b}$, F. Robinet ${ }^{11}$, A. Rocchi ${ }^{10 a}$, L. Rolland ${ }^{12}, \mathbf{R}$. Romano $^{6 a c}$, D. Rosińska ${ }^{17 g}$, P. Ruggi ${ }^{2}$, B. Sassolas ${ }^{13}$, D. Sentenac $^{2}$, R. Sturani ${ }^{4 a c}$, B.L. Swinkels ${ }^{2}$, A. Toncelli ${ }^{8 a b}$, M. 


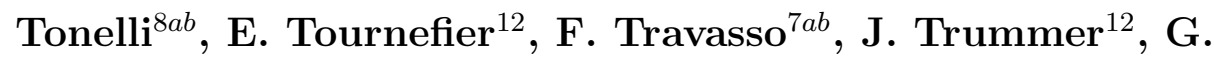
Vajente $^{8 a b}$, J.F.J. van den Brand ${ }^{14 a b}$, S. van der Putten ${ }^{14 a}$, M. Vavoulidis ${ }^{11}$, G. Vedovato ${ }^{16 c}$, D. Verkindt ${ }^{12}$, F. Vetrano ${ }^{4 a c}$,

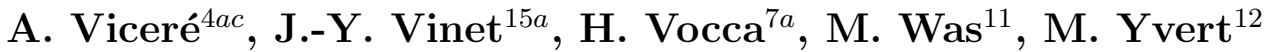

${ }^{1}$ AstroParticule et Cosmologie (APC), CNRS: UMR7164-IN2P3-Observatoire de Paris-Université Denis Diderot-Paris VII - CEA : DSM/IRFU

${ }^{2}$ European Gravitational Observatory (EGO), I-56021 Cascina (Pi), Italy

${ }^{3}$ ESPCI, CNRS, F-75005 Paris, France

${ }^{4}$ INFN, Sezione di Firenze, I-50019 Sesto Fiorentino ${ }^{a}$; Università degli Studi di Firenze, I-50121 ${ }^{b}$, Firenze; Università degli Studi di Urbino 'Carlo Bo', I-61029

Urbino $^{c}$, Italy

${ }^{5}$ INFN, Sezione di Genova; I-16146 Genova, Italy

${ }^{6}$ INFN, sezione di Napoli ${ }^{a}$; Università di Napoli 'Federico II ${ }^{b}$ Complesso

Universitario di Monte S.Angelo, I-80126 Napoli; Università di Salerno, Fisciano, I-84084 Salerno ${ }^{c}$, Italy

${ }^{7}$ INFN, Sezione di Perugia ${ }^{a}$; Università di Perugia ${ }^{b}$, I-6123 Perugia,Italy

${ }^{8} \mathrm{INFN}$, Sezione di Pisa ${ }^{a}$; Università di Pisa ${ }^{b}$; I-56127 Pisa; Università di Siena, I-53100 Siena ${ }^{c}$, Italy

${ }^{9}$ INFN, Sezione di Roma ${ }^{a}$; Università 'La Sapienza ${ }^{b}$, I-00185 Roma, Italy

${ }^{10}$ INFN, Sezione di Roma Tor Vergata ${ }^{a}$; Università di Roma Tor Vergata ${ }^{b}$, Istituto di Fisica dello Spazio Interplanetario (IFSI) INAF ${ }^{c}$, I-00133 Roma; Università dell'Aquila, I-67100 L'Aquila ${ }^{d}$, Italy

${ }^{11}$ LAL, Université Paris-Sud, IN2P3/CNRS, F-91898 Orsay, France

${ }^{12}$ Laboratoire d'Annecy-le-Vieux de Physique des Particules (LAPP), IN2P3/CNRS, Université de Savoie, F-74941 Annecy-le-Vieux, France

${ }^{13}$ Laboratoire des Matériaux Avancés (LMA), IN2P3/CNRS, F-69622 Villeurbanne, Lyon, France

${ }^{14}$ National institute for Subatomic Physics, P.O. Box 41882, 1009 DB ${ }^{a}$; VU

University Amsterdam, De Boelelaan 1081, $1081 \mathrm{HV}^{b}$, Amsterdam, The Netherlands ${ }^{15}$ Université Nice-Sophia-Antipolis, CNRS, Observatoire de la Côte d'Azur, F-06304

Nice ${ }^{a}$; Institut de Physique de Rennes, CNRS, Université de Rennes 1, 35042 Rennes ${ }^{b}$; France

${ }^{16}$ INFN, Gruppo Collegato di Trento ${ }^{a}$ and Università di Trento ${ }^{b}$, I-38050 Povo, Trento; INFN, Sezione di Padova ${ }^{c}$ and Università di Padova ${ }^{d}$, I-35131 Padova; Italy ${ }^{17}$ IM-PAN 00-956 Warsaw ${ }^{a}$; F.of Phys. Warsaw Univ. 00-681Warsaw ${ }^{b}$; Astro. Obs. Warsaw Univ. 00-478 Warsaw $^{c}$; CAMK-PAN 00-716 Warsaw $^{d}$; Bialystok Univ. 15-424 Bialystok $^{e}$; IPJ 05-400 Swierk-Otwock ${ }^{f}$; Inst. of Astronomy 65-265 Zielona Gora $^{g}$, PL

${ }^{*}$ Cardiff University, Cardiff, CF24 3AA, United Kingdom

${ }^{* *}$ Eötvös University, ELTE 1053 Budapest, Hungary

*** University of Birmingham, Birmingham, B15 2TT, United Kingdom

E-mail: bas.swinkels@ego-gw.it

Abstract. The Virgo interferometer is one of the big observatories aimed at detecting gravitational waves. This report will describe the Virgot upgrades and the commissioning work performed between the first (VSR1) and the second Virgo Science Run (VSR2). Some first results of VSR2 will be discussed, which was recently started with a good duty cycle and a inspiral range for the detection of binary neutron-star inspirals of $10 \mathrm{Mpc}$. To conclude, an outlook will be given on some future upgrades of the detector. 


\section{Introduction}

Virgo [1] is one of the large-scale, Earth based interferometers aimed at detecting gravitational waves that are now operating $[2,3,4]$. It is located at the site of the European Gravitational Observatory in Cascina, close to Pisa. Many years of construction and commissioning finally resulted in the first Virgo Science Run (VSR1), in which data was acquired non-stop from May till October 2007. Analysis of the obtained data is still ongoing, but some first results have already been submitted for publication. The commissioning work carried out before and during VSR1 has already been reported earlier [5]. This report will focus on the the activities carried out since the end of VSR1 up to the beginning of the second Virgo Science Run (VSR2), which started in July 2009.

Immediately after VSR1, about half a year was spent on the optimization of the control system, the mitigation of scattered light problems and some minor upgrades. After that, the interferometer was shut-down for about half a year to install some major upgrades in the injection system, the electronics and infrastructure, which were part of the so-called Virgo + upgrade. In parallel to the upgrades, a lot of work was spent to recover from a vacuum failure. After that, another half year was spent on commissioning the interferometer at high input power and to prepare for VSR2. The rest of this report will discuss the various upgrades and some relevant commissioning activities, some first results of VSR2 and will finally give a short overview of planned future upgrades.

\section{Detector upgrades}

\subsection{Vacuum incident recovery}

In May 2008, the Virgo interferometer suffered from a serious failure in its vacuum system. While pumping out the North-End tower after work inside the tower, one of the vacuum view-ports used for the local-control system imploded, which basically sandblasted the mirror with glass fragments. Fortunately, the big valve connecting the tower to the long arm was still closed, so damage was limited to a single payload and some minor parts of the tower.

The root cause of the failure has been found in a weak design of the view-port, which leads to high stress on the glass/metal interface. Using finite-element analysis and destructive testing, a much safer model of view-port has been identified. As a precaution, close to 100 existing windows in the interferometer have been replaced with this safer model. A new payload was constructed using a spare mirror, which was already polished but still needed to be coated. Although the recovery costed considerable time and effort, the incident had only limited effect on the the commissioning schedule since it happened shortly before a long, planned shut-down to installing various upgrades.

When the interferometer could finally be restarted, some clear changes in its working point were observed. The contrast defect had worsened by a factor 3 and a bi-stability of the control system at the beginning of the lock-acquisition had disappeared. Finally, 
the main modulation frequency had to be changed by as much as $700 \mathrm{~Hz}$ to recover a good sensitivity. The cause of these changes has not been understood completely, but might be related to the slightly different properties of the new North-End mirror.

\subsection{Injection system}

The main focus of the Virgo+ upgrade was the increase of the laser power sent into the interferometer. This was done to improve the sensitivity at high frequencies, where shotnoise dominates. For this purpose, a new laser amplifier was installed that can deliver up to $60 \mathrm{~W}$, of which $25 \mathrm{~W}$ remains at the input of the interferometer. A new premode-cleaner was installed in a small vacuum tank on the laser bench. This is a short triangular cavity that helps reducing the laser intensity noise at high frequencies (around the modulation frequency of $6 \mathrm{MHz}$ ) and performs spatial filtering of the beam before it is sent into the vacuum system. A remotely tunable in-vacuum Faraday Isolator was installed on the suspended injection bench. This allows the fine-tuning of its rejection ratio, which changes due to thermal effects in vacuum [6]. Finally, the end-mirror of the triangular, $144 \mathrm{~m}$ long input mode cleaner was changed with a heavier one. This should cure some problems in the control of the mirror, which suffered from radiation pressure effects that would have gotten worse with the increase of power.

\subsection{Thermal compensation system}

One issue that gets more critical with the increase of input power is absorption of the input test masses. This leads to thermal lensing, which can prevent a stable operation of the interferometer. The chosen solution is to use a thermal compensation system (TCS), which consist of annular-shaped beam of a high-power CO2 laser that are projected onto the input test masses. This shape creates a negative thermal lens that compensates the effect of the main beam [7]. To always keep the mirrors at the same temperature, also a central heating beam was implemented, which should replace the heat of the normal laser beam in case of an unlock.

When the system was first installed, evidence was found that the intensity noise of the CO2 lasers can couple to the gravitational wave signal, probably via a thermoelastic effect in the mirrors. To solve this problem, a power stabilization system has been installed recently. First results show a reduction of the noise by a factor of 10 .

\subsection{Phase camera}

One new diagnostic tool that was installed is the so-called phase-camera, which was inspired by a similar device used at LIGO [8]. In this system, one of the optical beams coming out of the interferometer is scanned over a pinhole-detector. Using a complex demodulation scheme, the optical phase and amplitude can be obtained for the carrier and both modulation side-bands individually. From these signals, phase and amplitude images can be reconstructed in software. The camera was used extensively for the 
fine alignment of the TCS, for the characterization of the mirror absorption and for determining the thermal lensing of the input test masses as a function of TCS power.

\subsection{Other upgrades}

During the shut-down, an important part of the electronics was replaced. This was done partly to replace obsolete parts and partly to gain a lot in processing power. New real-time optical fiber links were installed, which can be used to send any signal to any place within the experiment. Some of the real-time processors were changed from propriety systems to commodity PC's running real-time Linux. Most importantly, the analog-to-digital converters used for acquiring the photo-diode signals were upgraded from 16-bit to 18-bit systems.

Finally, a lot of invasive maintenance on the infrastructure has been performed during the shut-down, such as a complete re-wiring of the power distribution cables and the replacement of tubing for the heating system. Some machines were replaced or doubled to provide greater redundancy. The speed of the air-conditioning machine was reduced to lower the seismic and acoustic noise in the experimental hall.

\section{Commissioning activities}

\subsection{Control noise reduction}

The longitudinal motion of the mirrors of the interferometer is controlled in 4 degreesof-freedom, of which the differential length of the long cavities (DARM) contains the gravitational wave signal. Since there is a non-zero cross-coupling between the different degrees of freedom, noise in the auxiliary control loops can pollute the important DARM loop. This problem has been solved with a noise feed-forward technique [9]. Further reduction of the noise has been achieved by using control filters with optimized highfrequency roll-off, careful balancing of actuator responses and finding the lowest-noise signals for controlling the auxiliary degrees of freedom.

Similarly, the noise of the alignment system have been optimized by choosing the lowest noise error signals, optimizing the control filters, improving the electronics and covering the optical benches to reduce air-turbulence. The coupling of alignment noise to the longitudinal degrees-of-freedom has been reduced by careful centering of the beam on the mirrors. Both longitudinal and alignment control noise are currently not limiting the sensitivity above $10 \mathrm{~Hz}$, see Fig. 1.

\subsection{Scattered light}

Once the control noise was reduced, it became apparent that the sensitivity at low frequencies was limited by scattered light. This can form a problem when spurious beams hit a seismically excited component and then scatter back into the main beam. The phase modulation of even a tiny amount of scattered light is enough to spoil the 


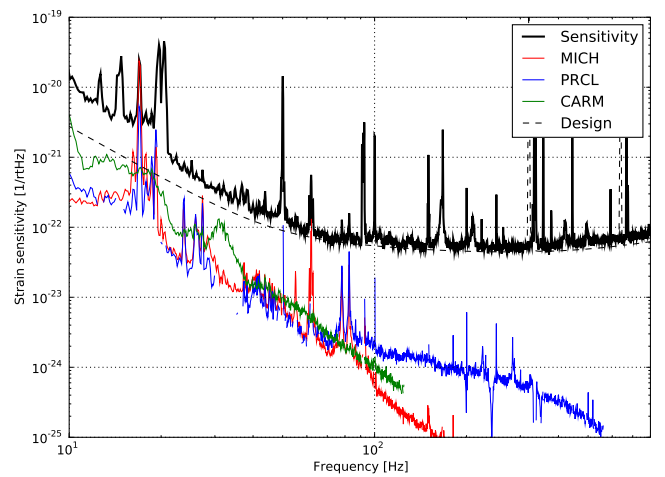

(a)

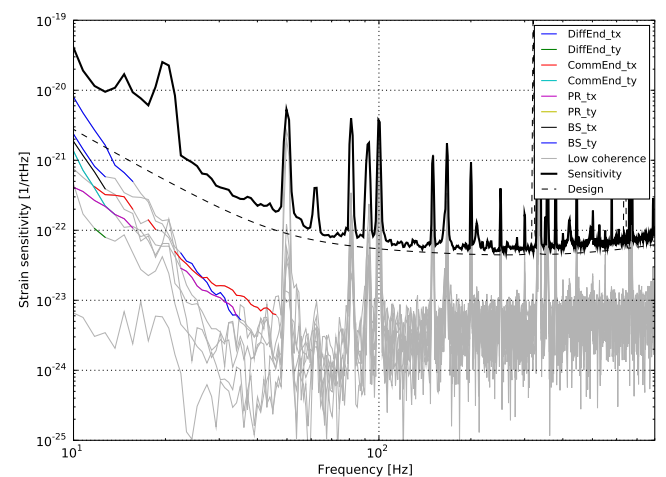

(b)

Figure 1: Projection of control noise to the sensitivity (a) Auxiliary longitudinal degrees of freedom (MICH, PRCL and CARM) (b) Angular loops

optical signal that probes the length between the seismically isolated test-masses. This problem was largely reduced by careful dumping of spurious reflections on the optical benches, by cleaning of optical components and by the damping of mechanical resonances of optical mounts and supports of optical benches. In case of high micro-seism, however, this noise can still dominate the low-frequency sensitivity.

\subsection{High power operations}

A large part of the commissioning time was dedicated to operating the interferometer with more input power. The power was increased in several steps from $8 \mathrm{~W}$ during VSR1 to $17 \mathrm{~W}$ now. Many parameters of the lock-acquisition procedure had to be retuned, but no major problems with the stability of the lock were encountered. The increased thermal lensing of the input test masses has been compensated using the thermal compensation system. Some control loops of the alignment system did become more critical due to radiation pressure effects. A further increase of the input power was foreseen up to 25 Watt, but this was not possible due to thermal effects in the injection system, which caused problems in the mode-matching of the monolithic reference cavity.

2.3.1. Aberration free operation Experiments were carried out to increase the TCS power so far that the thermal effects in the input test masses are compensated totally, as if the interferometer were operated at low power. This cold state could be confirmed by a higher optical gain, a more ideal transfer function of the fast frequency stabilization loop and in the images of the phase camera, see Fig. 2. The required power to reach this state was about $5 \mathrm{~W}$ per mirror. At that time the noise of the TCS lasers was too high to use so much power without spoiling the sensitivity. The standard configuration is now around $1 \mathrm{~W}$ per mirror, which keeps the interferometer in a state similar to $8 \mathrm{~W}$ of input power without TCS. 

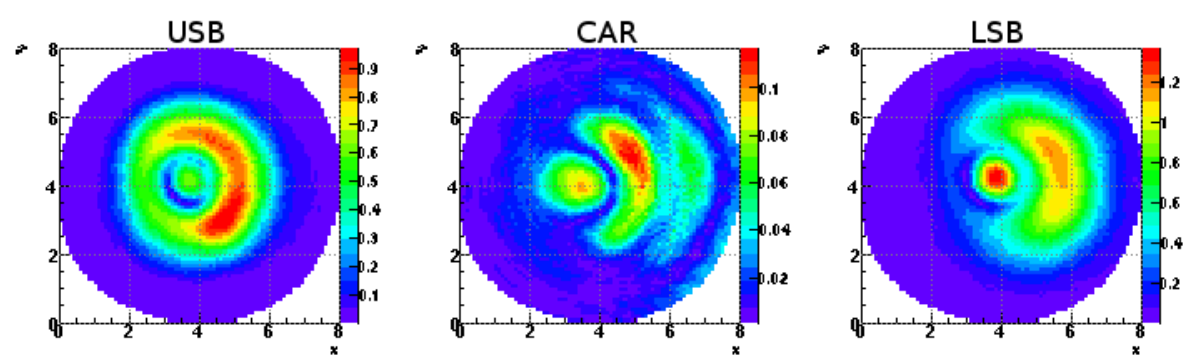

(a)
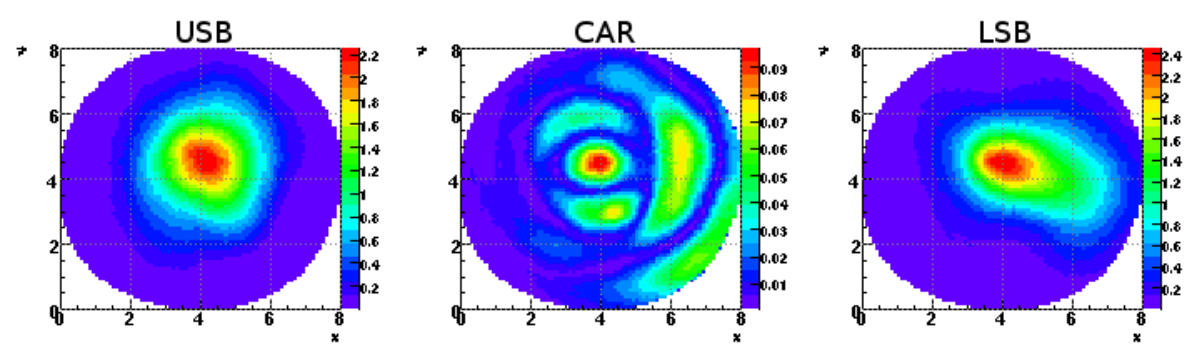

(b)

Figure 2: Images of the phase camera, showing the optical amplitudes of the upper sideband (USB), the carrier (CAR) and lower sideband (LSB) (a) without compensation of thermal lensing (b) with full compensation of thermal lensing

\subsection{Noise hunting}

Parallel to the other activities, there is a continuous campaign to find environmental noises that can couple to the gravitational wave signal. When such noise is discovered, a two-way strategy is employed, trying either to reduce the coupling or to reduce the noise (or both). In case of seismic noise, the coupling was reduced by mitigating scattered light as described in section 2.2. The seismic noise itself was reduced by replacing machines by less noisy ones or by seismically isolating the sources using springs. Another example is magnetic noise, which can couple to the magnets glued to the mirrors. The coupling has been reduced by replacing some magnets by weaker ones and totally removing those that were not used. The noise itself has been reduced by displacing some sources away from the main mirrors. A summary of all known noise sources is shown in Fig. 3.

\section{Second Virgo Science Run}

The second Virgo Science Run (VSR2) finally started on July 7 of 2009. An excellent robustness of the lock was achieved, with many locks lasting more than 50 hours, with a record of 143 hours. A science mode duty-cycle of over $90 \%$ was achieved during the first two months of the run. These numbers are mainly limited by weekly maintenance breaks and the occasional large earthquake, which causes one unlock per week on average. 


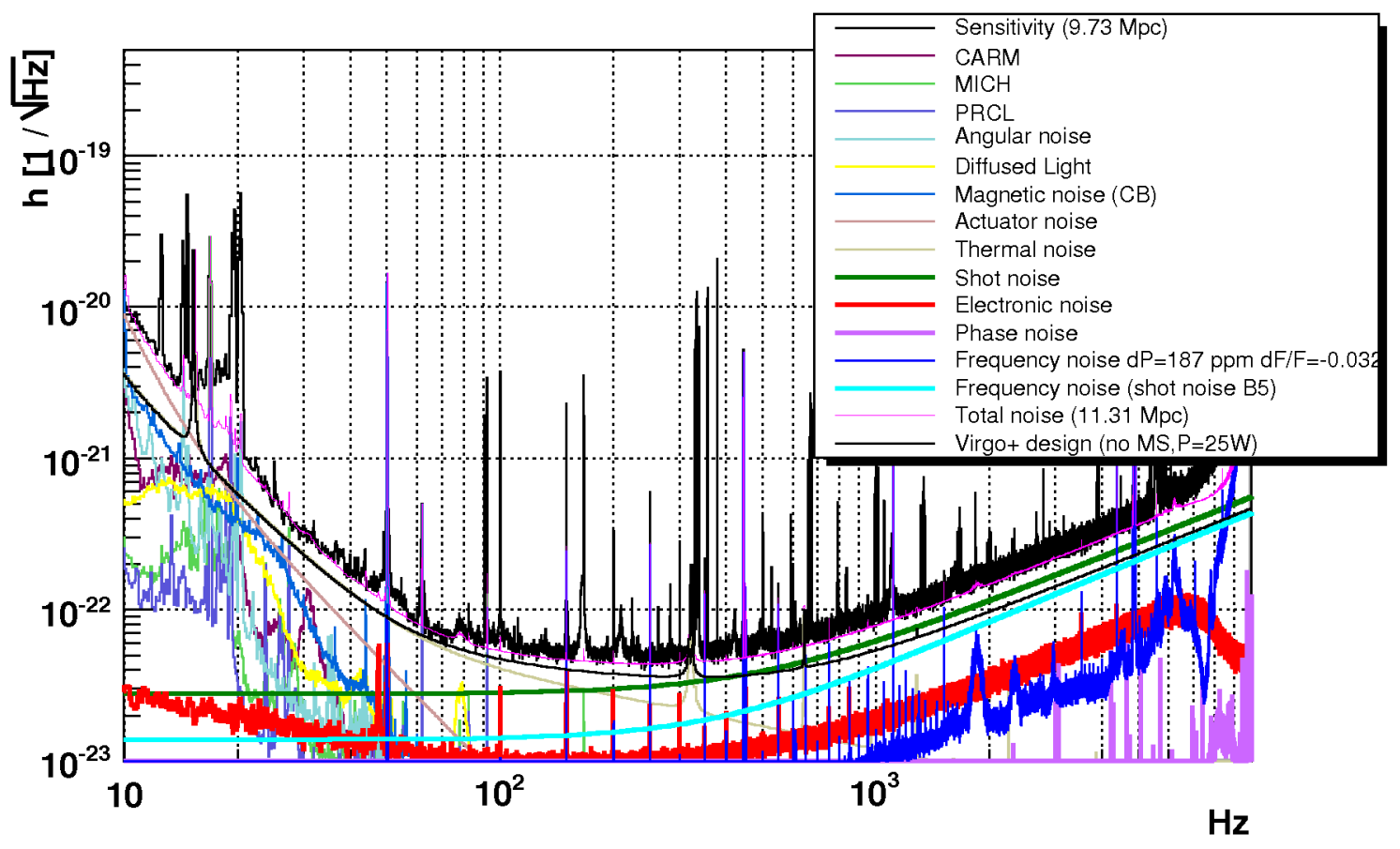

Figure 3: Noise budget of the Virgo detector during VSR2. The black curve shows the measured sensitivity, while the pink curve is the incoherent sum of all the known noises. At high frequencies, the sensitivity is above design due to increased shot-noise (less input power) and frequency noise (caused by the loss asymmetry of the arm cavities).

\subsection{Sensitivity and horizon}

The sensitivity of the Virgo detector has greatly improved over the last years, and is now close to its design. See Fig. 4 for the spectrum of the strain sensitivity of Virgo compared to those of the two LIGO detectors. Due to its advanced seismic isolation system [10], Virgo has currently the best sensitivity below $75 \mathrm{~Hz}$. Between 100 and 300 $\mathrm{Hz}$, the sensitivity of the LIGO detectors is a factor 2-3 better, which can be mainly explained by the difference in finesse of the arm cavities and longer length of the cavities ( $4 \mathrm{~km}$ for LIGO and $3 \mathrm{~km}$ for Virgo). Due to this difference trade-off, Virgo would be better suited to detect gravitational waves originating from inspiral events of heavier binaries and from low-frequency pulsars. The observed sensitivity is well understood, which is shown by the close match of the predicted and measured sensitivities in Fig. 3.

One of the main figures of merit of a gravitational wave observatory is the horizon or inspiral range, which is the distance to which an inspiral of binary stars with a typical mass can be observed with sufficient signal-to-noise ratio. During VSR1, this horizon fluctuated between 3 and $4.5 \mathrm{Mpc}$, while during the first months of VSR2, the horizon ranged from around 8 to a maximum of $10 \mathrm{Mpc}$, see Fig. 5. Note that the detection rate should scale with the volume of a sphere with a radius as large as the horizon, so for a similar observation period, the chance of detection a gravitational wave during VSR2 would be an order of magnitude larger than during VSR1. 


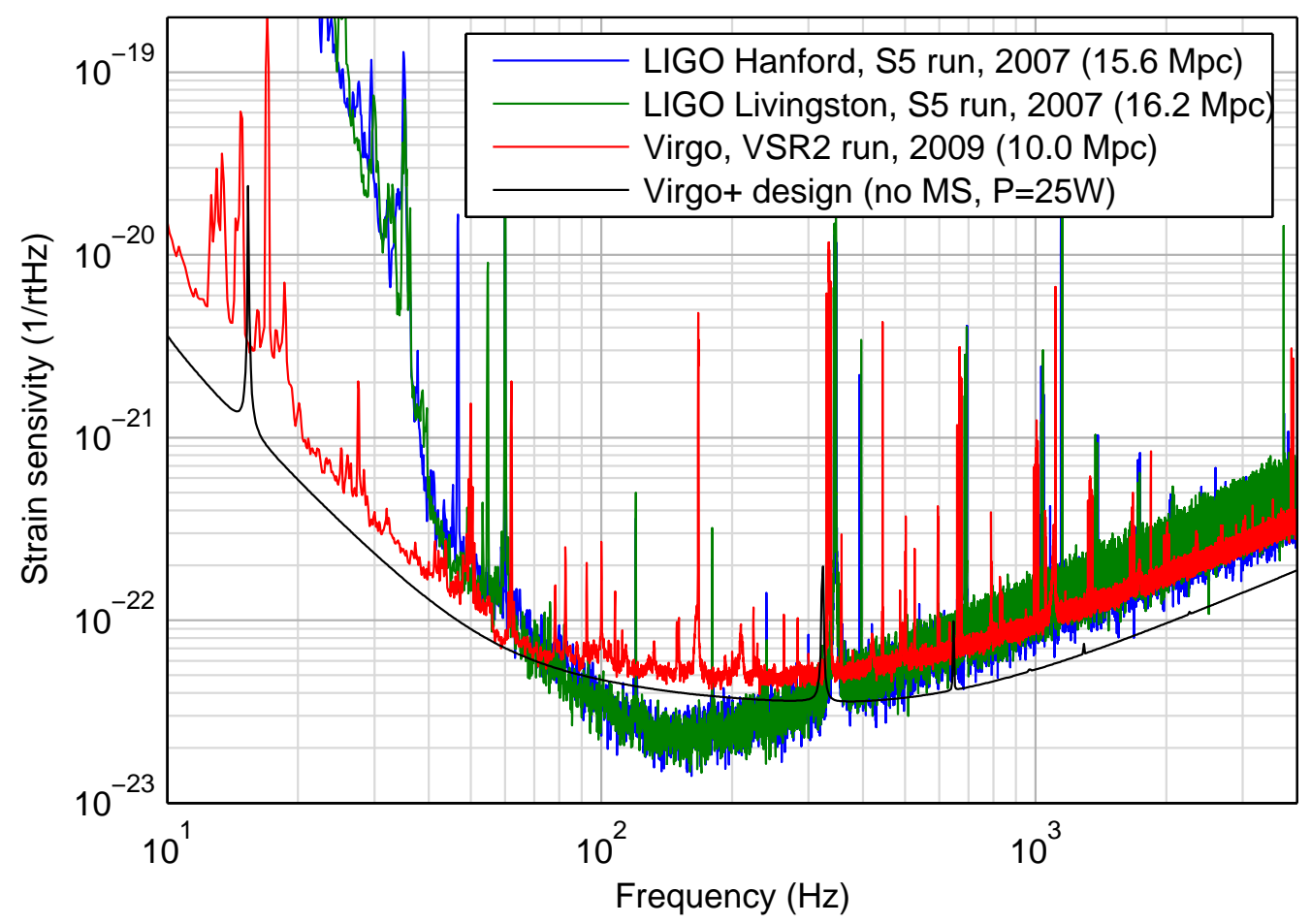

Figure 4: Strain sensitivity of the Virgo detector compared to the two LIGO detectors. Data courtesy of the LIGO Scientific Collaboration [11].

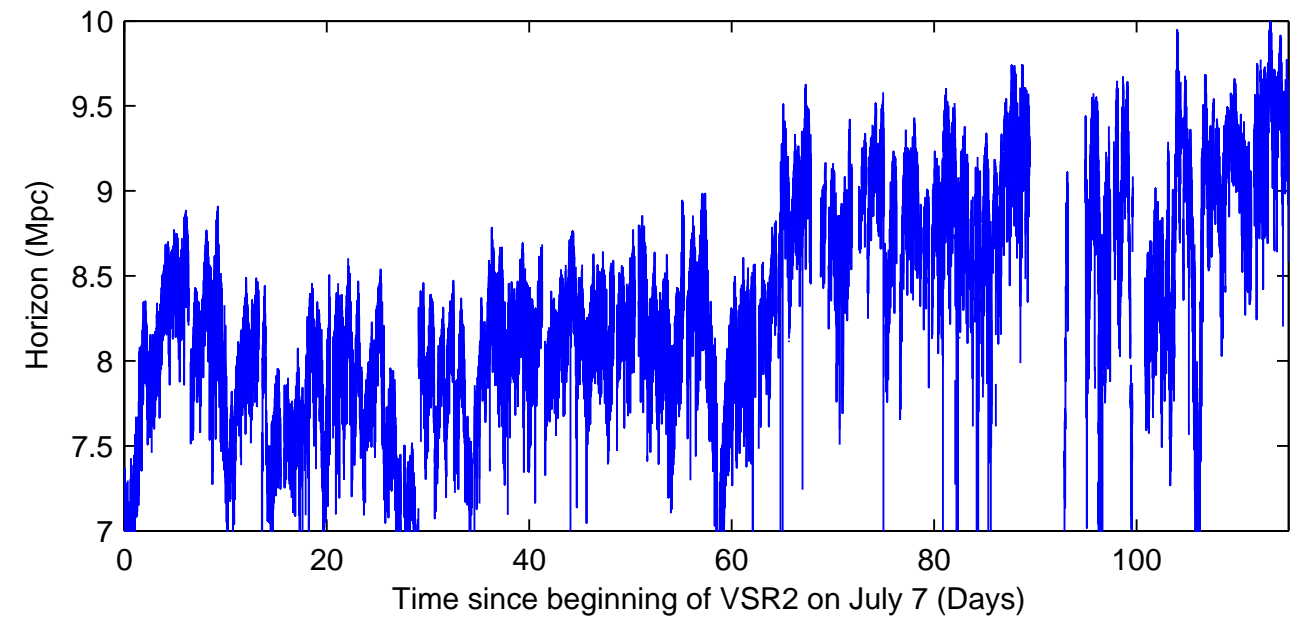

Figure 5: Evolution of the horizon distance for coalescing binary neutron star sources (averaged over the source sky position, inclination and polarization) during the start of VSR2 .

\section{Future upgrades of the detector}

In the immediate future, the science run will continue and only some small upgrades will be performed, such as the possible replacement of an output window with one that is 
anti-reflection coated. The next large upgrade will be the installation of new monolithic suspensions, which are new mirrors suspended by silica fibers. These fibers should have lower losses than the current steel wires, which should lower the thermal noise of the pendulum mode. The new coating should increase the finesse of the arm-cavities from 50 to 150. Together, these effects should give a substantial improvement of the sensitivity at low frequencies. Production of these new payloads has started recently, the installation is foreseen for early 2010. [12]

On a longer time scale, the Virgo collaboration is preparing for the realization of Advanced Virgo, for which a large design effort is ongoing. Advanced Virgo should have a sensitivity around 10 times better than the current interferometer. It will feature large upgrades in infrastructure, a further increase in input power, higher-finesse arm cavities, a new signal recycling mirror and heavier mirrors [13].

\section{Conclusions}

After its first science run in 2007, the Virgo interferometer underwent several upgrades and successfully recovered from a vacuum incident. After about a year of commissioning, a horizon for detecting binary star inspirals has more than doubled to $10 \mathrm{Mpc}$. Finally, in July 2009, Virgo started its second science run with good sensitivity and high duty cycle. Meanwhile, the installation of monolithic suspensions is being prepared for installation in early 2010 and a large design effort is ongoing for Advanced Virgo. These upgrades should provide a substantial gain in sensitivity, which would hopefully lead to the first detection of gravitational waves in the next few years.

\section{References}

[1] http://www.virgo.infn.it

[2] http://www.ligo.caltech.edu

[3] http://geo600.aei.mpg.de

[4] http://tamago.mtk.nao.ac.jp

[5] F. Acernese et al., CQG 25, pp. 114045 (2008), Status of Virgo

[6] F. Acernese et al., Appl. Opt. 47, pp. 5853-5861 (2008), In-vacuum optical isolation changes by heating in a Faraday isolator

[7] R.C. Lawrence, PhD Thesis, Massachusetts Institute of Technology (2003), Active wavefront correction in laser interferometric gravitational wave detectors

[8] K. Goda, D. Ottaway, B. Connelly, R. Adhikari, N. Mavalvala, and A. Gretarsson, Opt. Lett. 29, pp. 1452-1454 (2004), Frequency-resolving spatiotemporal wave-front sensor

[9] B. Swinkels, E. Campagna, G. Vajente, L. Barsotti and M. Evans, Virgo internal note VIR-0050A08 (2008), Longitudinal noise subtraction

[10] S. Braccini et al., Astroparticle Physics 23, pp 557565 (2005), Measurement of the seismic attenuation performance of the VIRGO Superattenuator

[11] Inspiral horizon distance and representative noise spectral density curves from LIGO's fifth and Virgo's first science run, in preparation

[12] The monolithic suspension for the interferometer Virgo, submitted to CQG

[13] The Virgo Collaboration, Virgo internal note VIR-0027A-09 (2009), Advanced Virgo Baseline Design 